\title{
Rare Case of Metastatic Colorectal Cancer to Uretral Meatus with Rapid Progression and Fatal Outcomes
}

\author{
Marwane Andaloussi Benatiya \\ Department of Urology, Hassan 2 Hospital, Agadir, Morocco \\ Zakaria El Asri \\ Department of Surgery, Hassan 2 Hospital, Agadir, Morocco \\ Ghizlane Rais \\ Medical Oncology Department, Regional Center of Oncology, \\ Agadir, Morocco
}

Doi: 10.19044/esj.2018.v14n21p12 URL:http://dx.doi.org/10.19044/esj.2018.v14n21p12

\begin{abstract}
Urethral metastases are extremely rare. A few cases of uretral metastasis in the literature have been found to arise from the colon. Because of the rarity of uretral metastasis and its similarity to primary uretral neoplasms, diagnosis is rarely evoqued. We report here a case of a 46-yearold female admitted to our hospital with complaints of rectal bleeding, bowel distension and urinary symptoms. Clinical examination revealed rectal mass associated with meatus urethral tumor. A biopsy yielded a diagnosis of rectal adenocarcinoma with metastasis to urethral meatus, and tumor cells were strongly and uniformly positive for CK20 and CDX2 at immunohistochemical analysis. A general examination has shown a sigmoid tumor with hepatic metastatic lesion. One month after diagnosis, the tumor grew rapidly, with hepatic invasion and therefore the patient was treated only by palliative care. She died from liver failure one month later. Although urethral metastasis originating from colon is rare, this entity is increasingly described. The synchronous character and the location of the metastasis in the uretral meatus, are two remarkable characteristics described in this case report. The purpose of presenting this case report is to raise awareness among clinicians to consider this clinical entity as a differential diagnosis when urethral mass is identified.
\end{abstract}

Keywords: Urethral metastasis, colonis origin

\section{Introduction}

Metastatic lesions to the urethra are extremelly rare (Fukata,2000). Urethral metastasis is less than $0.02 \%$ to all urological cancers with 
approximately 500 reported cases (Zhang,2015). Nearly $75 \%$ of the metastases originate from adjacent urogenital organs. The most common sites include the bladder and prostate (Chitale 2004, Karakose 2013). Metastasis to urethra from colorectal carcinoma is an extremely rare clinicopathological situation. It is frequently associated with widespread metastatic disease and poor prognosis (Chitale 2004, Karakose 2013). The synchronous character and the localisation of the metastasis in the uretral meatus, are two remarkable characteristics described in this case report.

\section{Case presentation}

A 46-year-old North African female was admitted to emergency department with complaints of bowel distension, rectal bleeding, and urinary symptoms such as gross hematuria and dysuria. Clinical examination revealed a rectal tumor at $6 \mathrm{~cm}$ from the anal margin and a tumor lesion at the urethral meatus burgeoning and bleeding (FIG 1). Computed tomography scan of the abdominopelvis showed a circumferential tumor of the sigmoide stenosing the lumen associated with a rectal tumor extended on $5 \mathrm{~cm}$ and one metastasis involving the liver. A high value of tissue tumor marker studies such as CEA and CA 19-9 was found.

After general anesthesia, preoperative sigmoidoscopy was made and revealed double tumor location on rectum and sigmoid. Cystoscopy found a unique lesion on the urethral meatus.

On laparotomy, a lesion occluding the lumen of the sigmoid was seen and discharge ileostomy was made. Rectal tumor and the lesion of urethral meatus was biopsied intraoperatively. Pathology of urethral specimen diagnosed adenocarcinoma consistent with colon metastasis. Immunohistochemical staining of urethral specimen showed tumor cells strongly positive for CK-20(fig 2), negative forCK-7 (Fig 3) and strongly positive for CDX2(Fig 4) which was consistent with colonic origin.

One month after diagnosis, the tumor grew rapidly, whether in rectal or urethral location with appearance of multiple liver metastasis. Patient was treated only by palliative care. She died from liver failure one month later. 
Figure 1. Exophytic growth to the urethral meatus.

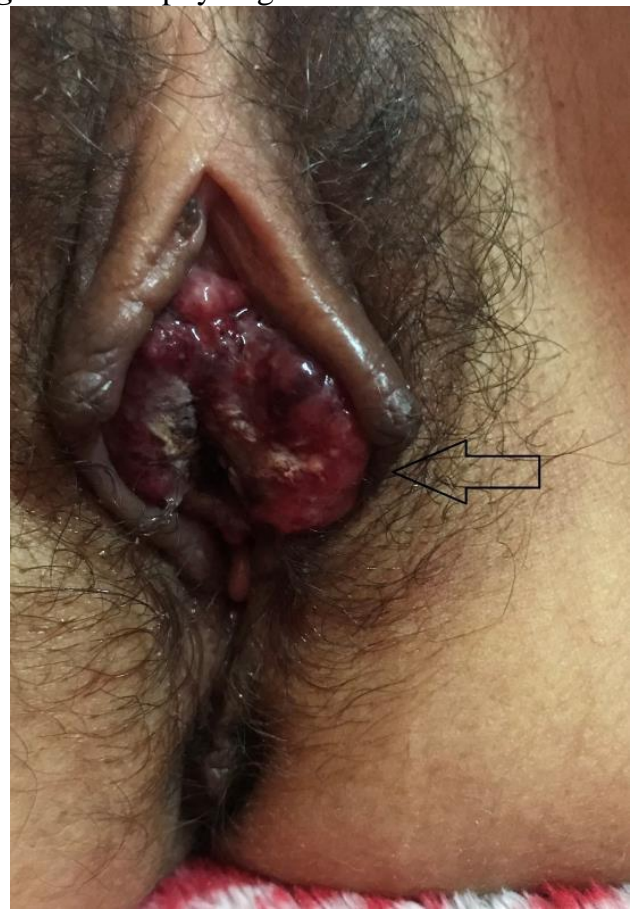

Figure 2. Immunohistochemical staining of urethral tumor shows a focal positivity of 
Figure 3. Immunohistochemical staining of urethral tumor shows lack of expression of CK7

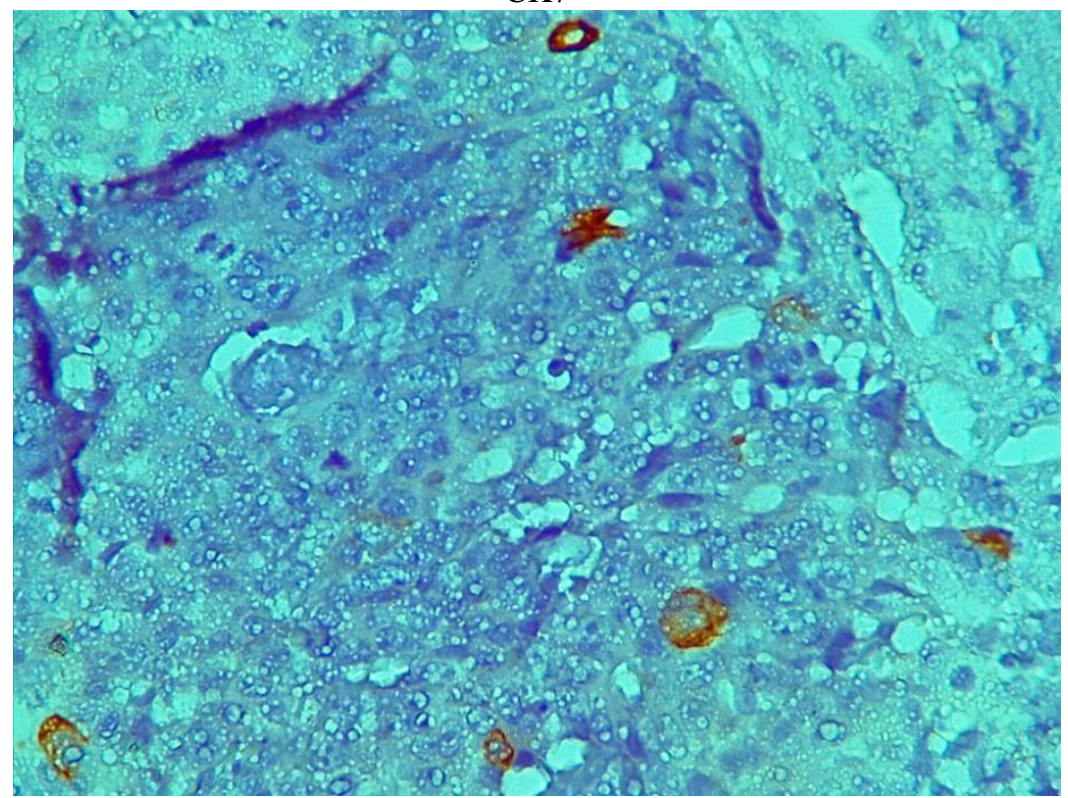

Figure 4. Immunohistochemical staining of urethral tumor shows diffuse expression of CDX2

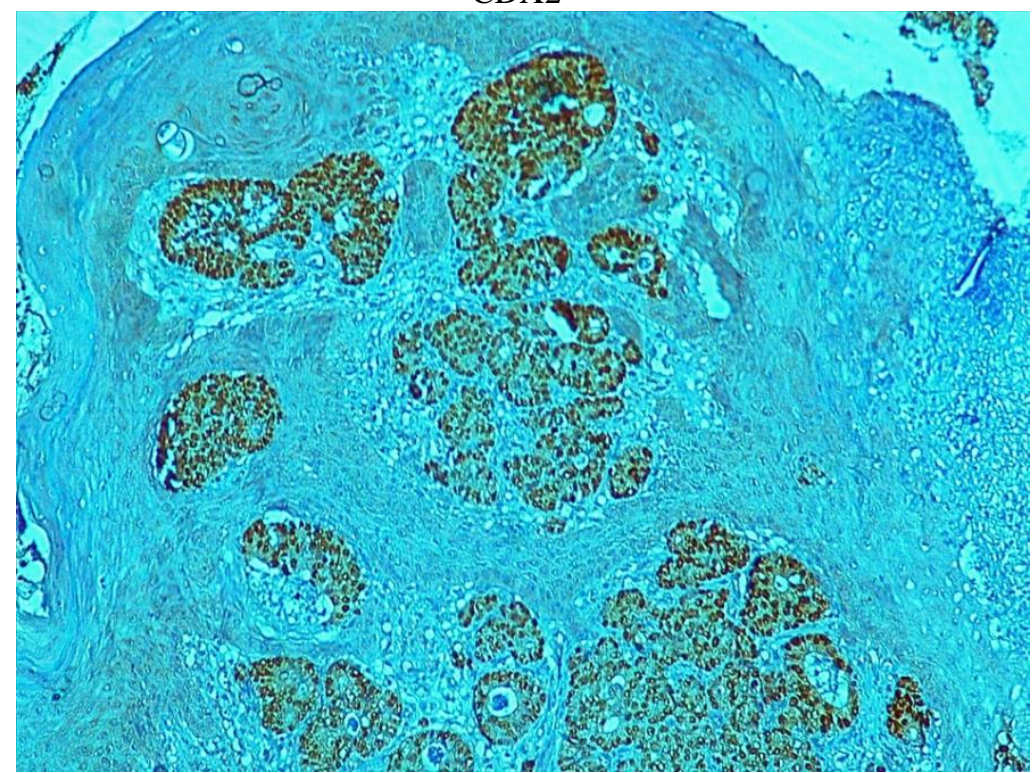

\section{Discussion}

Tumors of the urethra, whether primary or metastatic, are very rare. Cases of metastasis to the urethra have been documented in cancers of origin in the colon, the lung, the kidney and the prostate (Fukata 2000, Zhang 2015). Urethral metastases originating from the colon are extremely rare, and to the 
best of our knowledge there are less than 20 cases reported in the medical literature (Chitale 2004, Karakose 2013, Okaneya1991, Kupfer 1995)

The localization at the urethral meatus is even rarer.The bulbar urethra is the most affected part of the urethra in men (Fukata 2000). This is the first case reported in the english literature with involvement of the urethral meatus alone.

The interval between the appearance of the metastatic lesion and the primary tumor was 0.5-5 years (Fukata 2000). Most cases describing metachronous penile metastasis occur approximately 16-26 months after diagnosis and treatment of the colorectal primary (Chung 2008, Seo 2011). However we identified two cases of synchronous metastasis in the literature which were associated with disseminated disease (Chung 2008, Seo 2011).

The mechanism of dissemination is not well established, but there are several hypotheses: direct infiltration, lymphatic dissemination or hematogenous haematoma and the seeding of cancer cells through the urine.

The most common presentations and signs of urethral metastasis are dysuria, voiding dysfunction, perineal pain, priapism, penile nodules, mass, incontinence and urinary retention ( Karakose 2013, Chung 2008, Seo 2011).. Clinically, most symptoms in patients with urethral metastasis are urethral bleeding, hematuria and less acute urinary retention. Male patients sometimes have penile masses or perineal masses (Zhang 2015, Karakose 2013).

The most beneficial diagnostic tool are imaging studies such as CT, MRI and PET/CT. Magnetic resonance can be very useful for assessing local extension. Moreover, it is interesting to detect asymptomatic intraluminal urethral lesion. Because of the location of the metastasis on the urethral meatus in our patient, the diagnosis was made clinically and confirmed by pathology (Seo 2011, Kazama 2014). MRI was not performed in our patient because its general condition quickly deteriorated.

F-18 FDG PET/ CT can be useful for detecting the primary site of the metastasis and silent lesions when primary colonic tumor and urethral metastasis are metachronous. There is only two reported cases of man with sigmoid colon adenocarcinoma and synchronous metastatic lesions in urethra detected with PET-CT (Karakose 2014, Seo 2011) .

Immunohistochemical studies are essential to determine the origin of the primary tumor (LI 2004). Pathologists should be aware of the possibility of metastasis from the colon. In most reported cases, urethral tumor was 
considered to have metastasized from colon cancer pathologically by both morphological examination with hematoxylin and eosin staining and immunohistochemical Staining. In general, metastatic lesions are strongly positive for CK 20 and CDX2 and negative for CK 7 . The present case exactly showed the phenotype of CK20+/CK7-/CDX2+, suggesting that the urethral tumor was derived from primary tumor of sigmoid colon or rectum.

In general, patients with metastatic colonic cancer to the urethra have a poor prognosis because they are usually seen in advanced stages. Approximately $50 \%$ of patients with follow-up information are dead within 2 years following the urethral metastasis (Kupfer 1995, Kazama 2014)

Treatment of urethral metastasis from a colonic source must be undertaken via a multi-disciplinary approach. A multidisciplinary and individualized therapeutic approach should be carried out for each patient, depending on the symptoms and the extent of their disease. Treatment modalities are local surgery, systemic chemotherapy and local radiotherapy (Kazama, 2014). Local surgical excision is the treatment of choice for solitary urethral lesion (Okaneya,1991; Zhang 2015) but it should be made based on each patient's symptoms and the findings.

In the case of multiple metastasis, there is no evidence that there is a survival advantage from urethral resection, but resection of the urethral lesion is effective in preventing lower urinary tract complications such as retention (Karakose 2014, Chitale 2004, Kazama 2014). In general, patients are treated with palliative chemotherapy as recommanded for the treatment of metastatic colorectal cancer based on $5 \mathrm{FU}$, oxaliplatin, Irinotecan and biotherapy like Bevacizumab. Chemotherapy with Bevacizumab was planned for our patient with liver and urethral metastases but its general condition quickly deteriorated and was treated only with palliative care.

Radiotherapy was rarely used in urethral metastasis patient. However, it was useful in some cases for local control after surgery when the urethral metastasis was unique and resected.

\section{Conclusion}

Urethral metastasis from colorectal cancer is rare and indicates poorer prognosis. In case of urethral tumor, pathologists and urologists should be aware of the possibility of metastasis from the colon.Treatment of urethral metastasis from a colonic source must be undertaken via a multi-disciplinary approach. 


\section{Conflict of interest and source of funding}

No conflicts of interest to disclose.

\section{Consent}

Written informed consent was obtained from the patient's next of kin for publication of this case report and any accompanying images. A copy of the written consent is available for review by the Editor-in-Chief of this journal.

\section{Abbreviations}

CT: computed tomography; IHC: immunohistochemistry.

\section{Acknowledgement}

We would like to thank Dr. B.Mouaouia pathologist at Histopathology laboratory AGADIR, for her contribution to the images.

\section{References:}

1. Fukata S, Inoue K, Moriki T, Shuin T (2000) A solitary metastasis of renal cell carcinoma to the urethra. J Urol;163:1245-1246.

2. Zhang K, Da J, Yao HJ, et al.(2015) Metastatic tumours of the penis: A report of 8 cases and review of the literature. Medicine;94:132.

3. Chitale SV, Burgess NA, Sethia KK, Love K, Roberts PF (2004): Management of urethral metastasis from colorectal carcinomas. ANZ J Surg;74:925-927.

4. Ayhan Karakose, Ozgu Aydogdu, Yusuf Z. Atesci (2013) Unusual Urethral Metastasis from Colon Carcinoma Presenting with Difficult Urination and Hematuria. Curr Urol;7:152-154

5. Okaneya T, Inoue y, Ogawa A (1991) Solitary urethral recurrence of sigmoid colon carcinoma. Urol Int;47:105-107.

6. 6Kupfer HW, Theunissen P, Delaere KP(1995) Urethral metastasis from a rectal carcinoma. Acta Urol Belg;63:31-32.

7. Chung TS, Chang HJ, Kim DY, et al. (2008) Synchronous penile metastasis from a rectal carcinoma. Int J Colorectal Dis;23:333-4.

8. Seo HS, Kim ES, Kim S, et al (2011) A Case of Urethral Metastasis from Sigmoid Colon Cancer Diagnostically and Prognostically Indicated by F-18 FDG PET/CT. Nucl Med Mol Imaging;45:319-23.

9. Kazama et al (2014) Urethral metastasis from a sigmoid colon carcinoma: a quite rare case report and review of the literature. BMC Surgery 14:31.

10. Li MK, Folpe AL(2004) CDX-2, a new marker for adenocarcinoma of gastrointestinal origin. Adv Anat Pathol.;11:101-5. 